\title{
Analysis of radiation effects on chicken eggs and meat irradiated by nanosecond electron beams
}

\author{
Ruslan Vazirov ${ }^{1,2,1}$, Sergey Sokovnint ${ }^{1,2,3}$, Alisa Romanova $^{4}$ and Ksenia Moiseeva ${ }^{1,4}$ \\ ${ }^{1}$ Ural State Agrarian University, Yekaterinburg, Russia \\ ${ }^{2}$ Ural Federal University named after the first President of Russia B. N. Yeltsin, Yekaterinburg, \\ Russia \\ ${ }^{3}$ Institute of Electrophysics of the Ural Division of the RAS, Yekaterinburg, Russia \\ ${ }^{4}$ Ural Federal Agricultural Scientific Research Center of Ural Division of Russian Academy of \\ Sciences, Yekaterinburg, Russia
}

\begin{abstract}
Chicken and eggs are considered the most common product in the world. Microorganisms on the surface and inside these food products reduce the shelf life and may be the causative agents of the disease among consumers. To solve this problem various methods of thermal and chemical treatment are used, which have several disadvantages. Radiation processing is a modern method of suppressing contamination of bacteria and increasing the shelf life of food products. Unfortunately, this technology has a negative effect on the physico-chemical and organoleptic properties of the product. Therefore, for food and hatching eggs the dose level is limited about $3 \mathrm{kGy}$. However to solve this problem it is possible to use a low-energy electron beam. This approach allows irradiating with a disinfecting dose only the surface and a shallow depth of products. Experiments on the irradiation of chicken meat and eggs were carried out at the accelerators URT- 0.5 and URT-1. Studying the effect of irradiation with a nanosecond electron beam (NEB) on edible chicken eggs-showed no significant changes in the eggs structure and physico-chemical properties. This is primarily due to the low depth of electron penetration. Therefore, most-of the product is not irradiated by electrons. Thus, irradiation using the URT accelerators will reduce the main problem of the food radiation technology.
\end{abstract}

\section{Introduction}

Irradiation of food products (FP) is the processing of food with any kinds of ionizing radiation. This technology is used to control the content of pathogenic microorganisms, reduce microbiological contamination and insect infestation, suppress root germination and increase the shelf life of perishable products [1], [2].

Microbial contamination of chicken eggs and meat is a well-known phenomenon and has the serious negative economic consequences for the poultry industry [3]. Even in

1 Corresponding author: ruslan.vazirov@urfu.ru 
developed countries, the number of food poisoning outbreaks has increased, in which various Salmonella stamps have been involved [4]. Inadequate disinfection of eggs leads to about 230,000 cases of foodborne illness each year [5]. The most common procedure for the inactivation of microorganisms on the egg surface and in meat is chemical disinfection or heat treatment. However, treatment with ionizing radiation is an attractive alternative [6]. Currently, absorbed doses (AD) in the range from 5 to $25 \mathrm{kGy}$ are used for radiation processing to reduce the contamination by microorganisms or to increase the shelf life of food products.

However, a significant drawback of the radiation method of processing FP is the negative impact on the organoleptic and physico-chemical properties of FP. At the same time, radiation processing of eggs is limited because of the reduction in the quality of table eggs and the death of embryos in hatching eggs. Surface sterilization of raw eggs and meat with low-energy electron beam is an innovative method. Using the method of radiation surface disinfection will allow using the high doses for irradiation only the surface layer of food, limiting the internal exposure of products. This allows reducing the effects of irradiation on the quality of FP.

The surface disinfection using ionizing radiation (IR) sources can be described by the term of radiation surface disinfection (RSD). The RSD is the process of FP irradiation with a dose distribution, the maximum of which is located on the surface or at a small depth of the processed product.

A new and promising method of RSD is the use of a nanosecond electron beam (NEB) generated at URT-type accelerators [7]. This technology allows managing the distribution of the dose load across the depth of the processed product by changing the electron energy. This technology is applicable for RSD when the internal component of FP is not exposed or is exposed to small doses of IR, and, therefore, it is possible to minimize the negative effect of IR on the quality of products. Such an effect can be compared with the effect of increased background radiation. Consequently, the products processed by NEB cannot be equated to those irradiated with the "Radura" sign, which will allow avoiding a prejudiced consumer attitude and radiophobia.

\section{Material and method}

In this work, the effective depth of electron penetration and the AD of bremsstrahlung was calculated. All calculations were carried out in the PClab software package. The PCLab program is based on the EPHCA software package (electron-photon cascade), which is designed to calculate the spatial, energy, and angular distributions of electrons, photons, positrons, and protons in homogeneous and inhomogeneous substances. This program is suitable for determining the trajectory of electrons and positrons in the energy range of $10^{-2}$ $-10^{5} \mathrm{MeV}$, photons $-10^{-3}-10^{5} \mathrm{MeV}$. The calculations of the trajectories and the AD were made by simulating the interaction of the particle flux by the Monte Carlo method.

The experiments on the irradiation of chicken meat and eggs were carried out on the accelerator URT-0.5 and URT-1 [8].

To confirm the calculations, the dose was measured using plastic film and TLD dosimeters. The measurement of the $\mathrm{AD}$ on the surface was performed using film dosimeters $\mathrm{CO}$ AD (F) R-5/50. The optical density was measured using a spectrophotometer PE-5400VI at a wavelength of $512 \mathrm{~nm}$. To measure the distribution of bremsstrahlung $\mathrm{AD}$ inside the chicken eggs TLD-500 dosimeters were used. Dosimeters were located in sections of boiled chicken eggs (cut along or across) [9].

To determine the biological effect of NEB on FP, the changes of physico-chemical properties after irradiation were evaluated. For the analysis of the mass fraction of vitamin $\mathrm{A}$ in yolk and vitamin B2 in protein, a high-performance liquid chromatography (M-02- 
1006-08) was used. After irradiation, meat was analyzed according to the following regulatory documentation: $\mathrm{pH}$ determination - GOST R 51478-99; determination of the mass fraction of moisture - GOST 33319-2015; determination of the mass fraction of protein and nitrogen - GOST 25011-81; determination of the mass fraction of fat - GOST 23042-2015; determination of phosphorus mass fraction - GOST 32009-2013; determination of the mass fraction of chlorides - GOST R 51480-99; determination of amino acids - M-02-902-142-07.

A histological study of broiler meat was carried out after NEB irradiation.

The damaging effect of NEB on the tissues of hatching eggs and the emerging embryo was also investigated. Besides an ovoscopy, macroscopic analysis of the quality of hatching eggs were carried out. The incubation of hatching eggs using standard technology was used as in industrial poultry farming. The percentage of eggs hatching and the quality and health of young animals were evaluated daily during the rearing period before slaughter ( 37 days). The biochemical, immunological and hematological parameters of blood of broiler chickens were evaluated to identify possible metabolic and physiological disorders. After slaughter, the pathological-anatomical research of chickens from the experimental and control groups was conducted.

\section{Results}

The calculated effective path length for electrons with energies of $0.2,0.5$ and $1 \mathrm{MeV}$ are presented in Table 1 . The calculated values of the effective charge $Z=10$ and mass shell number $\mathrm{A}=20$ were used for the calculation.

$$
Z_{э \phi}=\sum_{i}^{n} Z_{i} \cdot a_{i}
$$

where $\mathrm{n}-$ the number of chemical elements in the substance;

$\mathrm{Z}_{\mathrm{i}}$ - the charge number of the element $\mathrm{i}$ in the substance;

$a_{i}$ - fraction of $i$ element atoms in the material.

$$
A_{3 \phi}=\frac{\sum_{i}^{n}\left(Z_{i} \cdot a_{i}\right)}{\sum_{i}^{n}\left(Z_{i} \cdot a_{i} / A_{i}\right)}
$$

where $A_{i}$ - mass number of element $i$ in the substance.

The calculations for meat for electrons with energies of $0.2,0.5$ and $1 \mathrm{MeV}$ are presented in Table 1. The parameters of the biological tissue equivalent were used for the calculations.

Table 1. Effective path-length of low-energy electrons in the food product.

\begin{tabular}{|c|c|c|}
\hline Energy, MeV & Eggshell, mm & Meat, mm \\
\hline 0.2 & 0.11 & 0.5 \\
\hline 0.5 & 0.46 & 1.9 \\
\hline 1.0 & 1.06 & 4.9 \\
\hline
\end{tabular}

Since all electrons are absorbed by the shell, the AD in protein and yolk is formed by bremsstrahlung. In the simulation, two sources of bremssrahlung were taken into account: from an aluminium foil (40 $\mu \mathrm{m}$ thick) of accelerator supporting the anode lattice (transparency $80 \%$ ) and from an eggshell. Total AD in yolk will be the sum of AD from all 
bremsstrahlung deposits. However, the main contribution to the AD from bremsstrahlung is the shell. When simulating in the PCLab program, the AD capacity was equal to $0,545 \cdot 105$ $\mu \mathrm{Gy} \mathrm{m} 2 / \mathrm{mA} \mathrm{h}$. Considering the distance from the shell to the center of the yolk $22.5 \mathrm{~mm}$, the $\mathrm{AD}$ from the bremsstrahlung of the shell equal to $0.03 \mathrm{cGy} /$ pulse was found. Thus, the $\mathrm{AD}$ in the center of the yolk equal to the sum of the $\mathrm{AD}$ from both bremsstrahlung contributions is $0.11 \mathrm{cGy} /$ pulse.

The measured AD inside eggs did not exceed $0.31 \mathrm{cGy} /$ pulse (average $0.18 \pm 0.05$ $\mathrm{cGy} /$ pulse), and in yolk - no more than $0.2 \mathrm{cGy} /$ pulse. At the same time, the AD of the electron beam on the surface of the egg was $0.2 \mathrm{kGy} /$ pulse. [10]. Consequently, with AD $=5 \mathrm{kGy}$, accumulated in 25 pulses and sufficient for disinfecting the egg surface from Salmonella, the AD of bremsstrahlung in protein and in yolk will not exceed 8 cGy and 5 cGy, respectively. These results well correlate with the simulation in the PClab software package.

The results of the content of vitamins in chicken eggs after irradiation with NEB AD $=5 \mathrm{kGy}$ are presented in Table 2. Indicators of the concentration of vitamin B2 in protein after irradiation with NEB exceed similar values for eggs from the control batch of table eggs. At the same time indicators of the concentration of vitamin A in yolk after irradiation with NEB does not change. The properties of the shell after irradiation with $5 \mathrm{kGy}$ AD also did not change. [9].

Table 2. Vitamin content in an egg.

\begin{tabular}{|c|c|c|}
\hline Type of analyses & Control, $\mathbf{~ g / m l ~}$ & $\mathbf{5} \mathbf{~ k G y , ~} \mathbf{~ m g} / \mathbf{m l}$ \\
\hline Vitamin A in yolk & $2.79 \pm 0.10$ & $2.83 \pm 0.10$ \\
\hline Vitamin B2 in protein & $7.06 \pm 0.31$ & $3.55 \pm 0.42$ \\
\hline Vitamin A in yolk & $2.79 \pm 0.10$ & $2.83 \pm 0.10$ \\
\hline
\end{tabular}

Table 3. The changes in parameters in broiler chickens after NEB treatment with bremsstrahlung radiation of $40 \mathrm{cGy}$.

\begin{tabular}{|c|c|c|}
\hline Parameter & Control group & $\begin{array}{c}\text { Experimental } \\
\text { group }(40 \mathrm{kGy})\end{array}$ \\
\hline The average weight of the carcass, $g$ & 1280 & 1305 \\
\hline The average mass of the pectoral muscles, $g$ & 383 & 390 \\
\hline Mass and ratio of red and white meat, $\mathrm{g}$ & 815 & 840 \\
\hline Concentration of hemoglobin (median), g / 1 & 51 & 56 \\
\hline $\begin{array}{l}\text { The number of red blood cells: mod (median), } \\
1012 / 1\end{array}$ & 2.9 & 3.4 \\
\hline The average weight of the carcass, $g$ & 1280 & 1305 \\
\hline
\end{tabular}

In the study of physico-chemical properties of chicken meat, no significant changes in the experimental samples compared to the control ones were found. Tendencies in the change of parameters up or down with increasing the dose from 2 to $10 \mathrm{kGy}$ were also not detected. Radiation surface disinfection of chicken meat by NEB with a dose of $2 \mathrm{kGy}$ did 
not lead to significant changes in the mass fraction of moisture, protein and fat. With an increase in $\mathrm{AD}$ treatment, there is a slight increase in the proportion of fat. However, it was found that in meat samples collected on the surface layers (up to $2 \mathrm{~mm}$ ), treated with NEB with $\mathrm{AD}=5 \mathrm{kGy}$ and above, there is a damage of cellular proteins, which is manifested by stratification of myofibrils, fibrillation of fibers, as well as disruption of the cell membrane structure - sarcolemma. In addition, signs of protein damage in the surface layers of muscle cells indirectly indicate that the dose of NEB has been reached on the surface of the meat, which provides damage to the protein in bacterial cells. It essentially confirms the antibacterial effect of NEB treatment. Considering the extrapolated mileage of electrons, the percentage of the mass of chicken meat processed with AD with NEB can be determined. Determining the amount of irradiated products, only $10-12 \%$ of the mass of chicken meat is exposed to NEB. In general, these changes in the surface layers of meat under the influence of NEB over $5 \mathrm{kGy}$ should not have a negative effect on product quality, since the depth of electron penetration, and accordingly, the share in the total amount of affected tissues is extremely small.

To determine the biological effect on the embryo, large doses were selected in order to register some changes, since the effect of small doses is difficult to detect. As a result of the experiments, it was found that chickens from eggs subjected to NEB treatment showed higher activity in behavioural responses than chickens from the control group. No significant effect of stimulation or suppression of chicken hatching was detected after the treatment of eggs with NEB with the dose in yolk of $40 \mathrm{cGy}$. In the experiments, the percentage of hatching in the experimental group was $96 \%$, in the control group - $94 \%$. The results of measurements of the physiological parameters of broiler chickens are presented in table 3. However, it should be noted that in the experimental group the period of chicken breeding was less on average than in the control group (16-18 hours and 22-24 hours).

\section{Conclusion}

Calculations of effective depth of low-energy electron penetration allow determining the maximum depth of radiation processing. The results obtained allow choosing the optimal energy to create the distribution of the $\mathrm{AD}$ and the maximum of it on the surface.

The value of $\mathrm{AD}$ generated from bremsstrahlung during radiation processing does not exceed the level of small doses. This value of AD should not lead to changes in physicochemical and organoleptic properties, and does not lead to a negative biological effect in the treated incubated chicken eggs. Moreover, the obtained results and other data in the literature [11] allow concluding that the brood of chicks can be stimulated. The method of radiation surface disinfection has all the prerequisites for its use in the agricultural sector.

Acknowledgements: This work was supported by the Russian Science Foundation, project No. 16-16-04038. The authors would like to thank Balezin M.E for irradiation the samples.

\section{References}

1. Radiation processing of food products. Basic technical requirements, GOST 333392015, 2016

2. GENERAL STANDARD FOR IRRADIATED FOODS CODEX STAN 106-1983, 2003. 
3. R.M. Ulmann, "Introducing irradiated foods to the producer and consumer Peaceful uses of atomic energy," in Proceedings of the fourth international conference, Geneva, Switzerland, 299-308 (1971)

4. E.C. D. Todd, Journal of Food Protection, 59 (1), 82 -92 (1996)

5. N. S. Bufano, Food Technology, 54 (8), 192 - 197 (2000)

6. P. Pinto, R. Ribeiro, L. Sousa, S. Cabo Verde, M. G. Lima, M. Dinis, A. Santana и M.

L. Botelho, Radiation Physics and Chemistry, 71 (1-2), 33 - 36, (2004)

7. S. Yu. Sokovnin, Yu. A. Kotov, S. N. Rukin, G. A.Mesyats, Russian Journal of Ecology, 27 (3), 214-216, (1996)

8. Yu. A. Kotov, S. Yu. Sokovnin, M. E. Balezin, Instr. and Exp. Tech., 43 (1), 102-105 (Jan. 2000) DOI : 10.1007 / BF02759009

9. S. Y. Sokovnin, M. E. Balezin, R. A. Vazirov, O. R. Timoshenkova, A. S. Krivonogova, A.G. Isaeva, I.M. Donnik, Radiation Physics and Chemistry, 165, 108398 (Dec. 2019) DOI: 10.1016/j.radphyschem.2019.108398

10. S. Yu. Sokovnin, R. A. Vazirov, M. E. Balezin, "Surface irradiation of chicken eggs by nanosecond electron beam," RAD Conference Proceedings, 2, 11-14 (2017) DOI: 10.21175/RadProc.2017.03

11. V.V. Pak "Reaction of the organism of chickens to the effect of ionizing radiation," Doctor of Biological Science dissertation, Moscow State Academy of Veterinary Medicine and Biotechnology named after K.I. Scriabin (Moscow, Russia, 2001) 